
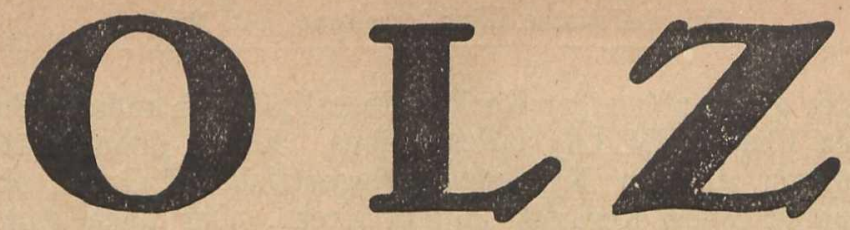

Oktober 1922

\section{Der Palast des Minos zu Knossos.}

Von Georg Karo.

Es ist ein seltener Fall von historischer Gerechtigkeit, daß Arthur Evans, der Jahre lang inmitten fast allgemeiner Skepsis die Existenz einer vorhistorischen Bilderschrift auf griechischem Boden verfochten hatte, die reichen Archive dieser Schrift im Palaste von Knossos entdecken durfte. Es ist ein noch selteneres Glück für unsere Wissenschaft, daß die märchenhaften Schätze dieses Königssitzes des III/II.Jahrtausends v. Chr. von einem Manne gehoben wurden, der Begeisterung, wissenschaftliche Akribie, Reichtum und wissenschaftliches Pflichtgefühl verband, und so nicht nur ausgraben und sammeln, sondern auch mit gewaltigen persönlichen Opfern die gefundenen Denkmäler restaurieren, erhalten und würdig veröffentlichen konnte. Wie viel durch die oft zu Unrecht geschmähten Bauarbeiten in den Ruinen von Knossos vor dem sicheren Untergang gerettet worden ist, kann ich bezeugen, da ich die Ausgrabungen seit ihrem Beginn im Jahre 1900 fast jedes Jahr besucht habe. Den damals erschienenen vorläufigen Fundberichten und den wichtjgen Einzelpublikationen (British School Annual VIff. Prehistoric Tombs of Knossos; The Tomb of the Double Axes at Knossos; Scripta Minoa I.) hat Evans nun den ersten Band ${ }^{1}$ eines monumentalen Werkes folgen lassen, das die Entwicklung nicht nur des Palastes von Knossos, von den ältesten Zeiten bis zum Ende der von ihm Mittelminoisch (MM.) genannten Periode, sondern auch mittelbar die der ganzen altkretischen Kultur in diesem Zeitraum darstellt. Ein ungemein wertvolles, reich und gediegen illustriertes Werk, das auch in Deutschland vielfach verbreitet ist, dank der weisen Auffassung von wissenschaftlicher Solidarität, die der Verfasser auch während des Krieges nicht verleugnet hat. Da das Buch weit über den Kreis der engeren Fachlente hinaus großes Interesse besitzt, sei eine eingehendere Angabe des Inhalts gestattet.

Evans gibt in seiner Einleitung (S. 1-31) eine kurze Charakteristik der altkretischen Kultur und seines in drei dreigeteilte Perioden ge-

1) The Palace of Minos at Knossos. Vol. I. The Neolithic and Early and Middle Minoan Ages. (XXIV, 721 S., 11 Tafeln, $542 \mathrm{Abb}$. und mehrere z. T. farbige Beilagen im Text.) 40. London, Macmillan \& Co. 1921. 2.6.6.-

377 gliederten chronologischen Systems. Die Eigenart dieser Kultur, ihre Beziehungen zum nahen Orient und Ägypten werden kurz skizziert, ebenso die zur Kykladen-Kultur des III/II. Jahrtausends und zur mykenischen" auf dem griechischen Festlande. Es folgt (S. 32-55) eine Schilderung der neolithischen Kultur, die auf Kreta der eigentlich minoischen vorausgeht und eine Vorstufe zu dieser bildet. Eine Reihe von Miniaturgefäßen und tönernen Idolen bezeugen die religiösen Bräuche dieser frühen Zeit, die übrigens im Rahmen des Neolithischen schon entwickelt und kaum sehr alt ist. Unter den gravierten Mustern auf Scherben und Spinnwirteln erscheinen schon Vorläufer der minoischen Pflanzenbilder, manche erinnern sogar an spätere Schriftzeichen. Sehr lehrreich ist das gelegentliche Fortleben der primitiven Idoltypen bis in ganz späte Zeit (SM. III). Überhaupt schließt sich an die neolithische Periode die Frühminoische (FM. I-III, S. 56-126) ohne scharfen Bruch an, wie denn auch die drei Phasen dieser ersten großen Kulturepoche Kretas ein Ganzes bilden und ineinander übergehen. Trotzdem sich die einzelnen Phasen nicht immer reinlich scheiden lassen, wäre es falsch, sie einfach zu verwischen und das ganze Frühminoische zu einer Einheit zusammenzufassen. Wo Knossos hier versagt, helfen Funde aus dem östlichen Kreta, besonders die Gräbér von Mochlos und die Hausruinen von Vasiliki, weiter. Höchst merkwürdige hochfüßige Becher, deren Verzierung gemasertes Holz nachahmt, kommen in zwei Höhlen vor, deren Inhalt noch ans Neolithische zu grenzen scheint. Aus einer entsprechenden Schicht in Knossos stammt ein ägyptisches Kugelgefäß aus Syenit (S. 65, Fig. 28), das vor- oder frühdynastisch sein muß. Nachbildungen und Nachwirkungen solcher ägyptischer Steingefäße sind in den Gräbern der nächsten Phase (FM. II, vor allem Mochlos und Messara) neben echt kretischen Formen nicht selten. Die hohe Vollendung dieser Väschen und des ihnen z. T. nachgebildeten, z. T. echt keramisch erfundenen Tongeschirrs, sowie der schönen Schmucksachen und Petschafte lassen es durchaus glaublich erscheinen, daß schon im FM. II vielzimmrige Häuser, geradezu kleine Paläste vorkommen (Vasiliki, vielleicht auch Palaikastro). Idole aus der Messara beweisen, daß damals die Tracht schon der späteren minoischen glich (für die Männer bloß ein Schurz, 
für die Frauen ein langer glockenförmiger Rock, S. 84). In der nächsten Phase (FM. III) fallen die stärkeren Beziehungen zu den Kykladen auf, die Anfänge der polychromen Keramik mit weißen und roten Mustern auf Firnisgrund und die höchst interessanten Petschafte von Stein und Elfenbein, von denen Evans eine Reihe bisher unbekannter abbildet (S. $117 \mathrm{ff}$.). Die Freude an der unbefangen kühnen Darstellung von Pflanze, Tier und Mensch äußert sich hier schon in überraschender Weise, auch vor mehrfigurigen Bildern schrecken die frühminoischen Künstler nicht zurück. Im Anschluß daran beginnt sich auch eine Art primitiver Ideogramme zu entwickeln, wenn auch von einer Schrift noch nicht die Rede sein dürfte. Selbst im Rahmen dieser raschen Entwicklung ist es aber doch verblüffend, daß sich die Herren von Knossos schon damals ein gewaltiges, mindestens $16 \mathrm{~m}$ hohes unterirdisches Gewölbe im weichen Felsen des Palasthügels aushöhlen ließen, an dessen Wandung eine Treppe in einer Art von Blendgalerie emporführte. Diese vom Südeingang des späteren Palastes überbaute Anlage ist auf S. 105 zum ersten Male abgebildet. Ihre Deutung ist noch nicht einwandfrei gelungen. Eine Zisterne scheint sie nicht zu sein. Evans erkennt darin einen monumentalen, befestigten Zugang zum Palaste, was mir um so weniger überzeugend scheint, als Befestigungen sonst überall im minoischen Kreta fehlen - ein besonders merkwürdiger Beweis für die einheitliche, hochentwickelte staatliche Organisation der Insel, die offenbar schon seit sehr früher Zeit eine starke Flotte vor auswärtigen Feinden schützte (tönerne Kähne schon FM. I, S. 57, Segelschiff auf einem Petschaft FM. III, S. 118).

Mit MM. I (S. 127-202) läßt Evans, the Age of Palaces" beginnen, gibt aber selbst $\mathrm{zu}$, daß schon die mächtigen Bauten des ausgehenden FM. diesen Namen verdienen würden. Ein Bruch ist nirgends festzustellen, bloß eine nun rascher fortschreitende Entwicklung. Die gewaltigen Palastanlagen in Knossos erlauben eingehende chronologische Erforschung, die Evans und sein getreuer Iolaos Duncan Mackenzie in musterhafter Weise durchgeführt haben. Durch eine Unzahl von Schichtengrabungen, Nachprüfungen und Einzeluntersuchungen von Mauern, aus deren Verbande Scherben gezogen wurden, ist ein förmliches Archiv von Scherben und anderen Kleinfunden aus allen Teilen und Schichten des Palastgebiets gesammelt und in Knossos selbst sauber geordnet aufbewahrt worden, wie es m. W. für keinen anderen antiken Bau in ähnlicher Vollständigkeit vorliegt. Nur die Ausgräber selbst verfügen natürlich über eine erschöpfende Kenntnis dieses Archivs, obwohl sie es mit vorbildlicher
Liberalität auch anderen Spezialforschern geöffnet haben. Es ist daher müßig und unrecht, wenn gelegentlich auf Grund oberflächlicher Arbeiten in Kreta, oder auch ohne diese, die Resultate von Evans und Mackenzie leichthin beiseite geschoben werden (z. B. L. Franchet bei J. Hazzidakis, Tylissos à l'époque minoenne, vgl. OLZ 1922, 286); ganz klar werden wir erst sehen, wenn uns die Erforscher von Knossos die endgültige Analyse dieser schwierigen baulichen und zeitlichen Probleme geben. Dazu ist das vorliegende Buch auch bloß eine Vorarbeit. Ihr wichtigstes Resultat für MM. I ist der bündige Nachweis, daß sowohl die große westliche Fassade des Palastes wie seine Ausdehnung nach Norden und Süden, und mit geringen Einschränkungen auch nach Osten, schon damals der späteren entsprachen, ebenso die Verteilung der einzelnen Flügel des weitläufigen Bauwerks um den großen Mittelhof und die Lage der Eingänge (vgl. den Plan nach S. 202). S. $131 \mathrm{ff}$. wird eine sehr lehrreiche Übersicht der älteren Steinmetzzeichen gegeben und ihre Bedeutung erörtert. Eine turmartige Anlage mit tiefen ausgemauerten Gruben (S. $136 \mathrm{ff}$.) scheint dem Anfang von MM. I anzugehören, wenn sie nicht gar dem sonderbaren Gewölbebau im Süden gleichzeitig ist. Auch diese merkwürdige Anlage ist noch keineswegs befriedigend erklärt. Vor allem bliebe noch zu untersuchen, ob Evans mit der Annahme gesonderter insulae innerhalb einer Festungsmauer recht hat. Er führt zum Vergleich das Heiligtum auf dem Gipfel des Iuktas (südlich von Knossos, beschrieben S. 151ff.) an, das in griechischer Zeit als Grab des Zeus galt und auch in den Anfang des MM. hinaufreicht. Aber auf solch' hoher steiler Bergkuppe brauchte ein bescheidenes kleines Heiligtum doch nicht befestigt zu werden, und andere Gründe können hier wie in Knossos die Stärke der Mauern bedingt haben.

Auch die bewunderungswerte Kanalisation des Palastes durch ein System sich verjüngender Tonrohre hat Evans schon für diese frühe Zeit nachgewiesen (S. $141 \mathrm{ff}$.). Im Grabritus vollzieht sich eine ebenfalls schon gegen Ende von FM. III angebahnte Wandlung: an Stelle der Familienoder Stammesgrüfte früherer Zeit (große Kuppelbauten im mittleren, rechteckige Einfriedungen oder hausähnliche Gräber im östlichen Kreta) treten rechteckige Gruben oder Kämmerchen und auch Einzelbestattungen in Töpfen oder Tonsärgen (Larnakes, S. 126. 150).

Ganz besonders lehrreich ist die Behandlung der Keramik dieser Phase, in die neben dem Fortleben älterer Typen das Aufblühen der bunten sog. Kamaresware fällt. Thre ersten Stadien werden auf Grund von Schichtengra- 
bungen klargestellt, wenn sich auch natürlich die Grenzen nach oben und unten nicht immer scharf ziehen lassen. Eine Reihe bisher unpublizierter wichtiger Stücke zeigt die Nachahmung verschiedener bunter Steinsorten in Ton, ferner die wachsende Freude an naturalistischen Darstellungen von Pflanzen und Tieren (vgl. vor allem die Schale aus Palaikastro mit plastisch aufgesetzter Herde und Hirten, S. 181, die knossische Schale mit bunt aufgemalten Fischen zwischen Felsen, S. 182, dann auch die merkwürdigen Rhyta in Gestalt von Stieren, an deren Hörnern kleine Männchen hängen, die ersten Abbildungen der dann so überaus häufigen Stierspiele, S. $188 \mathrm{ff}$.). Neben einem silbernen Becher aus Gurnia stehen getreue tönerne Nachbildungen (S. 192). Die ersten Petschafte mit sicherer Bilderschrift und Abdrücke von solchen erscheinen in sicher datierbaren Schichten, ebenso ein von Xanthudidis in einem Grabe von Platanos in der Messara entdeckter, hier zum ersten Male abgebildeter babylonischer Siegelzylinder mit der flehenden Ischtar vor Adad, der von Fachleuten um 2000 v. Chr. angesetzt wird (S. 198). Nicht minder wichtig sind ägyptische Skarabäen, die seit frühminoischer Zeit nicht gerade selten sind und auf Kreta Nachahmung finden (S. 199 ein schon bekannter echt ägyptischer Amethyst-Skarabäus mit minoischer Bilderschrift, S. 200 eine kretische Nachahmung, die eine ins Minoische umgestaltete Toeris trägt).

S. 203-314 umfassen die ältere Glanzperiode von Kreta, MM. II. Aus den einzelnen insulae, die Evans für MM. I annimmt, ist ein einheitlicher prunkvoller Palast geworden, dem nur noch der spätere Flügel der Privatgemächer fehlt (Plan vor S. 203). Auch der Thronsaal und die südlich angrenzenden Kulträume des Westflügels sind viel jünger, wir wissen nicht, was im MM. II an ihrer Stelle stand. Dagegen haben die Magazine und Wirtschaftsräume im wesentlichen schon ihre spätere oder eine ganz ähnliche Gestalt. Am steilen Abhang des Burghügels, östlich vom großen Hofe, müssen dem jüngeren vierstöckigen Prachtbau der Privatgemächer schon damals Anlagen vorausgegangen sein, wie ältere Mauerreste lehren. Der ganze Palast ist in Knossos wie in Phaistos sorgfältig nordsüdlich orientiert. Mächtige Außenmauern mit Orthostaten aus großen Blöcken kretischen Gipssteins, der dem Alabaster an Glanz fast gleichkommt, Hochbau aus Bruchsteinen mit eingezogenen Balken und Lehmverputz, seltener aus Lehmziegeln; Holzsäulen auf steinernen Basen, die in dieser Periode meist aus buntem hartem Gestein bestehen, entsprechend der frühminoischen Vorliebe für solche Steinarten, während später für Banglieder Kalk- oder Gips- stein, für Gefäße der weiche einfarbige Steatit fast ausschließlich verwandt werden. Sehr lehrreich ist die schrittweise Vervollkommnung der Kanalisation (S. 225 ff. mit Plänen und Schnitten), die nie wieder auf Kreta dieselbe Höhe erreicht hat wie MM. IIIII. Von den Heiligtümern wird unten noch zu sprechen sein.

Eine Menge wichtiger neuer Dinge bringt auch das Kapitel über die Keramik dieser Periode, der Blütezeit der Kamaresware (S. 231-270). Die gewaltigen Pithoi mit Reliefknöpfen und aus vollem Pinsel aufgeklatschten Firnisklecksen liegen in guten Abbildungen vor (S. $232 \mathrm{ff}$.), von der feinen bunten Ware gibt die Farbentafel I (vor S. 231) ausgezeichnete Proben der Übergangszeit von MM. I zu II, die noch die später aufgegebene Barbotine-Technik zeigen. Nachahmungen bunter Steingefäße treten auch in dieser Phase auf (S. 238), auch singuläre Stücke mit Reliefverzierung (täuschend lebenswahre Käfer S. 239) oder eingestempelten Ornamenten (S. 242, daselbst der vereinzelte verblüffende Fall einer eingestempelten Töpfermarke in Bilderschrift). Eine Auswahl z. T. unveroffentlichter Proben der schönsten, metallenen Vorbildern nachgeformten Eierschalen-Ware bieten Abb. $181 \mathrm{ff}$. und die Farbentafeln II. III (S. $241 \mathrm{ff}$.). Manche von diesen gehören ans Ende von MM. II, wenn nicht gar in den Übergang zu MM. III; die Angaben über die Schichtenverhältnisse auf S. $248 \mathrm{ff}$. sind für die Datierung von großer Bedeutung, aber manches bleibt natürlich noch unsicher. So würde ich dazu neigen, eine Reihe hier angeführter Vasen, unter anderen auch den prachtvollen, S. 254 zum ersten Male veröffentlichten Pithos mit Palmbäumen, für etwas jünger anzusehen. Mag er auch unter einer Mauer aus MM. III gefunden sein, so wissen wir doch nicht, ob diese nicht in den späteren Verlauf dieser Phase, der Pithos in ihren Anfang gehört. Die Frage ist wichtig, weil monumentale Vasenbilder wie dieses ohne Einfluß entsprechender Wandgemälde kaum denkbar sind. Evans hat demnach den Anfang der großen figürlichen Wandmalerei in Knossos schon im MM. II angesetzt, vor allem das älteste bisher bekannte figürliche Fresko, den Krokus- oder Safranpflücker, der nun endlich auf Tafel IV würdig abgebildet ist. Mir ist dieser zeitliche Ansatz immer zu hoch erschienen, ich schließe mich den Erwägungen Rodenwaldts in seinem vortrefflichen Buche: Der Fries des Megarons von Mykenai (S. 9. 63) durchaus an, ohne zu verkennen, daß gerade von diesem Gemälde viele Fäden zu MM. II zurückführen (Evans S. 264ff.). - Der Abschnitt über die Bilderschrift von MM. II, die schönen Petschafte und Siegelabdrücke, bietet gegen- 
über den erschöpfenden Ausführungen des Verf. in den Scripta Minoa nicht viel neues. Wertvoll ist die Zusammenstellung der im "Hieroglyphic Deposit" gefundenen Stücke und einiger bisher unbekannter (S. 277). - Die Fundumstände der bekannten Fayenceplättchen mit Häusern lassen leider eine genauere Datierung als MCM. II -III nicht zu (S. $301 \mathrm{ff}$.). Ob es sich hier wirklich um ein großes Mosaik einer belagerten Stadt handelt und die S. $309 \mathrm{f}$. abgebildeten Plättchen mit unkretischen Leuten, vielleicht Afrikanern, zugehören, scheint mir sehr zweifelhaft. - Negerköpfe erscheinen auf dem Fragment eines großen Stuckreliefs an einem goldenen Halsband in den Fingern eines Mannes(S.312.526).

MM. III beginnt nach Evans mit einer großen Umwälzung ${ }^{1}$, der die Paläste von Knossos und Phaistos zum Opfer gefallen sind. $\mathrm{Ob}$ fremde Feinde sie zerstört oder, wie Evans annimmt, eine neue Dynastie mit Gewalt die Herrschaft erobert hat, bleibt vorläufig ungewiß. Sicher hat aber kein Wechsel der Bevölkerung oder der Kultur stattgefunden, trotz mehrfachen Neuerungen, wie dem Ersatz der alten Bilderschrift durch die lineare, einigen Änderungen in Baukunst und Anlage der nel erbauten Paläste, die aber in allem wesentlichen den alten entsprechen, ja sogar große Teile von diesen einfach übernehmen. Der wichtigste Unterschied zwischen MM. II und III ist zweifellos die durchgreifende Herrschaft naturalistischer; oder besser impressionistischer Richtungen in der Kunst, die zwar seit dem Frühminoischen auf Kreta fühlbar waren, aber nun erst voll sich auswirken (S. 315-323). Die größte bauliche Leistung der neuen Zeit ist der Ostflügel der Privatgemächer, ein vierstöckiger Bau von raffiniertestem Luxus in der Anlage der Zimmer, Säulenhallen, verandaartigen Säle, Treppen und Lichthöfe. Evans beschreibt diesen Flügel ausführlich, mit reichem Abbildungsmaterial (S. 324 -359). Gerade hier haben die mühsamen und kostspieligen Stütz- und Ergänzungsarbeiten nicht nur den einzigartigen Bau vor der Zerstörung gerettet, sondern seine Erforschung und Erkenntnis erst möglich gemacht. Die neuen Pläne auf S. 328/9 zeigen dies deutlich, ebenso die Ansichten der großen Treppe in ihren verschiedenen Stadien. Von den in diesem Flügel gefundenen Freskenresten ist eines der wichtigsten ein labyrinthartiges Muster, das Evans S. $356 \mathrm{ff}$. mit entsprechenden ägyptischen und griechischen vergleicht.

Nördlich stoßen an die fürstlichen Privat-

1) Bisher hat man diese meist ans Ende von MM. III gesetzt, so auch Karo bei Pauly-Wissowa, Real-Enzykl. u. Kreta S. $1767 \mathrm{ff}$. Diese Frage bedarf noch weiterer Erörterung. gemächer ausgedehnte Wirtschaftsräume, zu denen besonders eine große Ölmühle mit Klärungs- und Ablaufkanälen und eine Reihe von Magazinen mit gewaltigen Pithoi gehören. Dieser Teil des Palastes war bisher ungenügend publiziert und bietet vielfache neue Aufschlüsse (S. 361-384). Von prächtigeren Räumen über den erhaltenen des Erdgeschosses zeugen zahlreiche Fragmente von Fresken und auch von einem lebensgroßen Stuckrelief mit Männern und Stieren, den längst bekannten Bruchstücken aus einer Halle im Norden des Mittelhofes verwandt. Leider sind nur kleine Brocken erhalten. Auch die Erkenntnis des Nordflügels ist gefördert worden, durch Erforschung einer Halle mit anstoßenden Magazinen im Osten des großen Nordeingangs, ferner der Pfeilerhalle und einer "Pfeilerkrypta", eines von sechs Pfeilern getragenen großen Kellerraumes, nördlich von jenem Eingang, der als Hauptverbindung des Palastes mit der Stadt Knossos solcher geräumiger Markthallen wohl bedurfte (S. 385-404). Westlich scheint eine Gruppe von Kulträumen, darunter eine kleine Säulenhalle und ein Badezimmer, an den Nordeingang gestoßen zu sein. Da hier der Weg zu dem theaterähnlichen Stufenbau im Nordwesten, wohl dem Hohen Tor des Palastes, führte, konnten jene Räume für Opfer und Reinigung bestimmt sein, wenn besonders vornehmer Besuch oder, wie Evans annimmt, Pilgerzüge ankamen (S. $405-422$ ).

Der Westflügel des großen Palastes ist für uns der wichtigste von allen, weil er die meisten Aufschlüsse über den Kult im alten Kreta gibt. Entgegen der griechischen Sitte geschlossener Tempelbauten scheint der minoische Kult sich im Innern der Häuser und Paläste abgespielt und mehrere kleine Räume umfaßt zu haben. In der königlichen Residenz gab es deren eine ganze Reihe, ihre Verwendung können wir leider im einzelnen noch nicht bestimmen. Evans legt mit Recht das größte Gewicht auf die Bedeutung des Doppelbeils als wichtigsten und häufigsten religiösen Symbols auf Kreta (S. 423-447). Immer wieder erscheint es auf Fresken, Gemmen und Petschaften, als Steinmetzzeichen auf Blöcken und Pfeilern, die sowohl tektonische wie religiöse Bedeutung haben, und auch in Bronze oder Stein als Kultobjekt, häufig auf steinernem Sockel und langem Schafte aufgepflanzt. Mehrere Beispiele sind hier zum ersten Male abgebildet, vor allem ein riesiges Doppelbeil aus Bronzeblech (Br. 1,20 m) das östlich von Knossos (bei Nirou Khani) in einem von Xanthudidis jüngst entdeckten Gebäude offenbar als anikonisches Kultbild diente (S. 436). Mit dem Doppelbeil verknüpft oder auch allein spielen Schleifen aus karrierten 
Stoffen eine Rolle im Kulte, sie finden sich auf Abbildungen sowohl wie in Nachbildungen aus Elfenbein und Fayence, auf Kreta wie auch in den Schachtgräbern von Mykenai (S. $430 \mathrm{ff}$.). Gegenüber der etwas unübersichtlichen Weiträumigkeit des großen Palastes bilden die Kultanlagen des sogenannten Südosthauses (S. $426 \mathrm{ft}$. zum ersten Male publiziert) ein geschlossenes Ganzes, wie denn überhcher Bauweise Häuschen ein Musterbeispiel minoist zierlich und fein, raffiniert und dabei erstaunlich winzig und eng.

An die Kulträume des Westflügels von Knossos schließen sich die ausgedehnten Magazine an, die endgültig in allen ihren Einzelheiten publiziert und besprochen werden (S. 448 -462). Durch genaue Erforschung der unterirdischen, steinernen Kästen, die hier die Stelle von Wandschränken einnehmen, hat Evans die zeitliche Entwicklung dieser Anlagen feststellen und eine für Kostbarkeiten bestimmte Gruppe von einer anderen scheiden können, in der offenbar die gewaltigen Ölvorräte der Fürsten von Knossos aufbewahrt wurden. Die Bedeutung des Öls für Kreta wird offenbar, wenn man erwägt, daß es gewiß der wichtigste Exportartikel im Verkehr mit Ägypten war.

Das vornehmste Heiligtum von Knossos war bekanntlich der großen Beherrscherin der Tierwelt g'eweiht. Die Gestalt ihres Kultbanes an der Westseite des Mittelhofes, südlich von der großen Treppe zum Obergeschoß, kennen wir nur aus dem Anfang der spätminoischen Zeit (Evans, Journal of the Royal Institute of British Architects 1911, 289). Ältere kostbare Weihegaben aus diesem Heiligtum sind uns zum Glück in zwei riesigen, längst bekannten unterirdischen Steinkisten erhalten geblieben. Von irdischen Stemple Repositories, wie er sie nennt,
diesen Tems (S. $463-523$ ) eine neue eingehende
gibt Evans (S Beschreibung und Abbildungen einiger bisher unveröffentlichter Stücke, so vor allem des schönen Fayence-Reliefs der Kuh, die ihr Kalb säugt, und einiger Siegelabdrücke (S. 696 ff.). In demselben Abschnitt findet man auch eine Farbentafel (Taf. V) des prunkvollen Spielbrettes aus dem Palaste und Abbildungen von Resten entsprechender Stücke aus Knossos und Mykenai (S. 471-485). Daran schließt sich eine lehrreiche Erörterung der altkretischen Fayencetechnik und ihrer Beziehungen zu Ägypten. Sehr willkommen ist auch die Publikation einiger köstlicher Tonreliefs mit Krabben und Muscheln auf felsigem Boden, die teils von großen Gefäßen stammen, teils wohl von richtigen Reliefbildern, wie die berübmten fliegenden Fische und Muscheln aus den Temple Repositories. Es ist klar, daß diese ganze Kleinkunst sich an die große Malerei anlehnt. Über deren Entwicklung im MCM. III gibt Seite 524-551 Aufschluß; darin eine Reihe neuer Abbildungen meist von kleinen Fragmenten: die oben erwähnten Finger eines Mannes mit einer Halskette mit goldenen Negerköpfen, das Bruchstück einer Volksmenge, Vorläufer der spätminoischen Miniaturfresken, wundervolle Lilienstengel (Taf. VI), die leider sehr geringen Reste einer Gruppe fast lebensgroßer Damen in blauen Gewändern (S. 545 von Gilliéron geistreich aber etwas ausgiebig ergänzt). Im übrigen bereitet Evans eine monumentale Gesamtpublikation der Fresken von Knossos vor.

Nach der neuen Einteilung kommt die $\mathrm{Ke}$ ramik in MM. III etwas schlecht weg, weil die kostbarsten Stücke schon der vorangehenden Phase zugeschrieben werden. Immerhin bleibt Interessantes genug, sowohl an grober und einfacher Ware, die massenhaft im Palaste aufbewahrt, wohl auch dort verfertigt wurde (S. 552 -590), wie auch an Pithoi und kleineren Gefäßen mit schöner weißer Bemalung. Darunter ragen vor allem die Töpfe mit Lilienstengeln hervor (S. $576 \mathrm{ff}$. und 603), andere mit Wicken (S. 606), auch ein paar große Pithoi mit Delphinen zwischen Felsen (S. 608, einmal weiß auf Firnisgrund, das andere in der neuen Technik der Firnisbilder auf Tongrund). Ein schönes Rhyton mit Palmbäumen (S. 594f.) hat Evans treffend als Nachbildung eines Straußeneies erkannt, wie sie ebenfalls als Rhyta montiert auch in den Schachtgräbern von Mykenai vorkommen. Eine tönerne Badewanne (S. 580) ist in sehr passender Weise mit Algen bemalt. Alle diese Darstellungen gehen natürlich auf Vorbilder der großen Malerei zurück, die gerade in dieser Zeit mit besonderer Vorliebe die reiche Pflanzenwelt der Insel wiedergibt. Daneben lebt die alte bunte Verzierungsweise von MM. II sich aus in pflanzlichen Ornamenten und Nachbildungen von Steingefäßen (S. 595 ff., Farbentaf. VII). Bedauerlich ist es, daß Evans noch immer die sogenannten Kamares-Scherben aus Mykenai, Tiryns, Orchomenos zum Teil für knossisch hält (S. $599 \mathrm{ff}$.), was sie ganz sicher nicht sind. Darin stimmen die kundigsten deutschen und englischen Forscher überein.

Die Beziehungen der Kleinkunst zur großen Malerei erörtert Evans im Anschluß an die Gemmen und Siegelabdrücke von MM. III. Da diese z. T. durch ihre Fundumstände datierbar sind, bilden sie ein besonders wertvolles Material. Eine Reihe neuer Abbildungen bereichern diesen Abschnitt (S. 669-700), an den sich wertvolle Bemerkungen über die eigenartigen geflügelten Fabelwesen und Mischgestalten der minoischen Kunst und ihre Beziehungen zu 
Ägypten anschließen. Die Erörterung der kretischen Linearschrift ist kurz gefaßt, da nur die kleine Klasse A in unsere Zeit fällt. Was hier gegeben wird (S. 612-646), steht schon im wesentlichen in den Scripta Minoa, ebenso die Bemerkungen zu dem berühmten Diskus mit Bilderschrift aus Phaistos (S. 647-668). Dieses einzigartige Denkmal ist zusammen mit einem Tontäfelchen der Klasse A und Scherben aus MM. III gefunden worden. Die eingestempelten Schriftzeichen, primitive Vorläufer der Druckschrift, unterscheiden sich durchaus von den kretischen, und ich stimme Evans in der Annahme bei, daß der Diskus nicht minoisch ist, sondern voraussichtlich aus Anatolien stammt. Wo man so schrieb, läßt sich noch nicht ermitteln. Die Verbindungen zwischen kretischer und vorderasiatischer, besonders hethitischer Kunst mehren sich. Schon in der frühminoischen Keramik und Glyptik finden sich auffällige Ahnlichkeiten mit Vasen aus der alten Hethiterstadt Ganesch (heute Kültepe) in Kappadokien, in der ja schon vor 3000 v. Chr. babylonische Kaufleute saßen (E. Meyer, Reich u. Kultur d. Chetiter S. 51 ff. Taf. 5, Weber, Hethit. Kunst S. 7; Originale in Berlin). Aus dem Ende des 3. Jahrtausends sind die vereinzelt in frühminoischen Gräbern gefundenen babylonischen Zylinder wichtig (außer dem oben erwähnten ein leider sehr zerstörter aus Mochlos). Die bekannte steinerne Sphinx aus Hagia Triada erklärt sich am leichtesten als Nachbildung eines babylonischen Vorbildes. Die Hethiter mögen hier die Vermittler gewesen sein. In den Palastbauten von Boghaz-köi hat Valentin Müller Beziehungen zur zeitgenössischen kretischen Architektur nachgewiesen. Hethitische Siegelzylinder sind auf Kreta und in dem spätmykenischen Schatzfunde von Tiryns aufgetaucht (Arch. Anz. 1916, S. 145). Aber die Schrift des Diskus von Phaistos ist keineswegs hethitisch, und man mu doch betonen, daß sämtliche orientalischen Fundstücke auf Kreta ein halbes Dutzend kaum übersteigen, während andererseits die einzigen minoischen Spuren in Vorderasien einige Scherben aus SM. I sind, die Herzfeld und Sarre in Samarra ausgegraben haben. Überhaupt ist ja die verblüffende Selbständigkeit der minoischen Kunst einer ihrer größten Vorzüge.

Sehr viel reicher sind die ägyptischen Funde auf Kreta und die kretischen in Ägypten; aber gerade sie, die uns die wichtigsten chronologischen Anhaltspunkte für die minoische Kultur geben, bereiten auch große Schwierigkeiten, da sich die Ägyptologen so wenig einig sind. Allgemein wird für den Beginn der XVIII. Dynastie der Anfang des 16. Jahrhunderts v. Chr. anerkannt, der mit dem Anfang der spätminoischen Periode zusammenfällt. Von hier bis zum Ende dieser Periode, um 1200 v. Chr., besteht keine Meinungsverschiedenheit, um so mehr für die Zeitspanne zwischen der XII. und XVIII. Dynastie. Neben den minoischen Scherbenfunden aus Kahun ist vor allem ein Grab von Abydos wichtig, das eine schöne bunte Vase aus dem Ende von MM. II enthält (Evans, S. 268, Suppl. Taf. IV). Leider ist hier nur der Teil des Grabinhaltes abgebildet, der sich im Ashmolean Museum befindet, nicht aber die beiden Zylinder Sesostris' II. (1903-1885) und Amenemes' III. (1846-1798), auf denen die Datierung des Grabes in die XII. Dynastie beruht; v. Bissing, der Abdrücke dieser Zylinder besitzt, teilt mir freundlichst mit, daß die Schreibung der Königṣnamen nicht die in der XII. Dynastie übliche sei. Auch sei es bedenklich, Zylinder, also Amulette, noch dazu zweier verschiedener Könige, zur Grundlage einer chronologischen Bestimmung zu machen. Für die Fayencegefäße, den Bronzespiegel, die Figürchen von Affen, Igeln und Nilpferden kann v. Bissing auch Beispiele aus dem Neuen Reich anführen; die Flaschen mit mehrfach eingezogenem Halse kämen in dieser Form in der XII. Dynastie noch nicht vor, wohl aber in der darauf folgenden Ubergangszeit. Endlich betont er, daß die ,third section" des Grabes, zu der die minoische Vase gehöre, gar nichts enthalte, was eine Datierung: ermögliche. Andererseits reicht die Blüte der Stadt Kahun bis zur Mitte des 18. Jahrhunderts herab. So bestätigt alles die Annahme von Evans (S. 290), daß die zweite mittelminoische Periode bis zum Ende des. 18. Jahrhunderts dauerte. Dagegen spricht keineswegs die in einer derselben Periode angehörigen Schicht des Palastes von Knossos gefundene ägyptische Statuette aus Diorit (S. $286 \mathrm{ff}$.), da ihre absolute Datierung, wie mir $\vee$. Bissing bestätigt, unmöglich ist. Die Statuette kann zu irgendeiner Zeit von der XII. bis zur XVIII. Dynastie entstanden sein, man braucht sie keineswegs zeitlich von dem Alabasterdeckel mit dem Namen des Hyksoskönigs Chian zu trennen, der ebenfalls in Knossos zutage kam. Evans, stets an Ideen reich, hebt hervor, daß die Zerstörung der Paläste von K 10 ssos und Phaistos mit dem Zusammenbruch cies ägyptischen Reiches zeitlich ungefähr zusammenfällt, und dann die Beziehungen zwischen beiden Ländern eine zeitlang nachlassen. Er weist (s $292 \mathrm{ff}$.) auf die uralten Hafenanlagen vor der Insel Pharos beim späteren Alexandria hin und erkennt in ihnen sehr geistreich eine minoische Anlage, einen Stützpunkt der kretischen Fürsten für ihren Verkehr mit Ägypten, entsprechend den spätern griechischen Kolonien. 
Diese Frage müßte an Ort und Stelle näher nachgeprüft werden.

Wenn man MM. II ins 18. und MM. I in die ersten beiden Jahrhunderte des 2. Jahrtausends setzt, wofür ja auch der erwähnte babylonische Zylinder spricht, so bietet das alles keine Schwierigkeiten (die Zeitangaben bei Fimmen, Die kretisch-myken. Kultur S. $152 \mathrm{ff}$. und Karo bei Pauly-Wissowa u. Kreta XI 1766 wären entsprechend zu berichtigen). Sehr störend sind dagegen die durch Steingefäße, Petschafte und Skarabäen in größerer Zahl unwiderleglich nachgewiesenen Beziehungen Kretas zu Ägypten in der frühminoischen Periode. Denn diese Stücke werden z. T. ins Alte Reich, z. T. sogar in vordynastische Zeit gesetzt, und nach dem neuen chronologischen System von Borchardt käme man damit sogar ins 5. Jahrtausend hinauf. Es erscheint mir vollkommen ausgeschlossen, daß die frühminoische Periode fast ebenso viele Jahrtausende gewährt haben sollte wie die Blütezeit der mittelminoischen Jahrhunderte. Dann müßten die Gräber sehr viel zahlreicher, die Fundschichten unendlich viel tiefer sein, während sie tatsächlich den mittelminoischen etwa entsprechen. Auch die ganze Entwicklungsart dieser Kultur mit ihrem raschen Aufstreben widerspricht durchaus einer so langen Dauer. Bei einzelnen Fundstücken, wie dem oben erwähnten Steingefäß vordynastischer Zeit aus Knossos, könnte man ja eine unbegrenzt lange Erhaltung annehmen; befinden sich doch ganz ähnliche vordynastische Steingefäße heute noch im Schatze von San Marco zu Venedig und im Evkaf-Museum in Konstantinopel, als christliche und islamische Kultgeräte haben sie die Jahrtausende überdauert. Aber die ägyptischen Fundstücke aus dem Alten Reich sind auf Kreta zu zahlreich, als daß man sie auf solche Weise wegerklären könnte. Und wenn auch eine Reihe von Stücken wesentlich jünger sein mögen, wie mir wiederum v. Bissing freundlichst nachweist, so wird auch dadurch das Problem nicht gelöst. Wir müssen hoffen, daß sich die Ägyptologen aufs neue mit diesen Fragen unter Berücksichtigung des kretischen Materials befassen werden. Evans aber gebührt für sein neues Werk der aufrichtigte Dank der gesamten Altertumskunde, nicht nur der klassischen. Möge der zweite Band dem ersten bald folgen. 